\title{
ON EXTREMAL PROBLEMS FOR PAIRS OF UNIFORMLY DISTRIBUTED SEQUENCES AND INTEGRALS WITH RESPECT TO COPULA MEASURES
}

\author{
FABrizio Durante ${ }^{1}$ - JuAn Fernández-SÁnCheZ ${ }^{2}$ \\ Claudio Ignazzi ${ }^{3}$ - Wolfgang Trutschnig ${ }^{4}$ \\ ${ }^{1}$ Dipartimento di Scienze dell'Economia, Università del Salento, Lecce ITALY \\ ${ }^{2}$ Grupo de investigación de Teoría de Cópulas y Aplicaciones, Universidad de Almería, La Cañada \\ de San Urbano, Almería, SPAIN \\ ${ }^{3}$ Dipartimento di Matematica e Fisica "Ennio De Giorgi", Università del Salento, Lecce ITALY \\ ${ }^{4}$ Department for Mathematics, University of Salzburg, Salzburg, AUSTRIA
}

ABSTRACT. Motivated by the maximal average distance of uniformly distributed sequences we consider some extremal problems for functionals of type

$$
\mu_{C} \mapsto \int_{0}^{1} \int_{0}^{1} F \mathrm{~d} \mu_{C},
$$

where $\mu_{C}$ is a copula measure and $F$ is a Riemann integrable function on $[0,1]^{2}$ of a specific type. Such problems have been considered in 4 and are of interest in the study of limit points of two uniformly distributed sequences.

\section{Communicated by Robert Tichy}

\section{Introduction}

According to [15], given a Riemann integrable function $F$ defined on $[0,1]^{2}$ and two uniformly distributed sequences $\left(x_{n}\right)$ and $\left(y_{n}\right)$ in $[0,1)$, a general problem

(C) 2020 BOKU-University of Natural Resources and Life Sciences and Mathematical Institute, Slovak Academy of Sciences.

2010 Mathematics Subject Classification: 11K06, $62 \mathrm{H} 05$.

Keywords: Uniform distribution, Copulas, Extremal problems.

Licensed under the Creative Commons Attribution-NC-ND 4.0 International Public License. 


\section{F. DURANTE - J. FERNÁNDEZ-SÁNCHEZ - C. IGNAZZI - W. TRUTSCHNIG}

is to find limit points of the sequence (see also [10])

$$
\left(\frac{1}{N} \sum_{n=1}^{N} F\left(x_{n}, y_{n}\right)\right)_{N \in \mathbb{N}} .
$$

As noted for instance in [4, 6] (see also [5]), this problem is equivalent to determining extreme values of the functional

$$
\mu_{C} \mapsto \int_{0}^{1} \int_{0}^{1} F \mathrm{~d} \mu_{C}
$$

over all possible copula measures $\mu_{C}$. Indeed, as shown in [6], this problem can be embedded in the general theory of mass transportation and optimal transport (see, e.g., 1, 11, 14]), and has important applications especially in risk management [13].

Here we focus on some results presented in [4] and [6] about extremes of functionals of type (10). Specifically, we present some generalizations of previous results by using different proof techniques. Although the general existence results could be also derived via optimal transport techniques [11, the present approach may provide an additional viewpoint to handle problems for uniformly distributed sequences.

\section{Main results}

We start by introducing some notations that will be used in the sequel.

If $\vartheta$ is a measure on the Borel $\sigma$-algebra $\mathcal{B}\left(\mathbb{R}^{2}\right)$ of $\mathbb{R}^{2}$ then $G_{\vartheta}$ denotes its measure-generating function defined by

$$
G_{\vartheta}(x, y)=\vartheta((-\infty, x] \times(-\infty, y]) .
$$

Vice versa, for every two-dimensional measure-generating function $G$ the corresponding measure on the Borel $\sigma$-algebra $\mathcal{B}\left(\mathbb{R}^{2}\right)$ will be denoted by $\vartheta_{G}$ or $\mu_{G}$.

For every one-dimensional distribution function $F$ the corresponding probability measure will be denoted by $\xi_{F}$ and $F^{-}$denotes the pseudo- (or left-) inverse of $F$. Given one-dimensional distribution functions $F_{1}, F_{2}$ the Fréchet class of $F_{1}, F_{2}$, i.e., the family of all two-dimensional d.f. with marginals $F_{1}$ and $F_{2}$, will be denoted by $\mathcal{F}\left(F_{1}, F_{2}\right)$.

Finally, the class of bivariate copulas will be denoted by $\mathcal{C}$. Two elements of $\mathcal{C}$ are the independence copula $\Pi, \Pi(x, y)=x y$, the comonotonicity copula $M$,

$$
M(x, y)=\min (x, y)
$$

and the countermonotonicity copula

$$
W(x, y)=\max (x+y-1,0) .
$$




\section{EXTREMAL PROBLEMS FOR PAIRS OF UNIFORMLY DISTRIBUTED SEQUENCES}

For every $H \in \mathcal{F}\left(F_{1}, F_{2}\right)$, Sklar's Theorem ensures that there exists a copula $C$, which is unique if $H$ is continuous, such that

$$
H=C \circ\left(F_{1}, F_{2}\right) \text {. }
$$

For more details, see 3 .

The following result generalizes both Theorem 4 in [4] and Theorem 3.6 in [6] in the sense that:

a) the integral is calculated over the product $I_{1} \times I_{2}$ of arbitrary intervals $I_{1}:=$ $\left[a_{1}, b_{1}\right]$ and $I_{2}:=\left[a_{2}, b_{2}\right]$ of $\overline{\mathbb{R}}$ with non-empty interior,

b) the marginal distributions may be discontinuous (see Corollary 2),

c) the integrand does not need to be smooth.

Additionally, the method of proof is new and is grounded on disintegration of the copula measure (see [3]). It should also be noticed that the first part of the Theorem has been essentially proved, in a different setting, in [11, Theorem 3.1.2].

Theorem 1. Suppose that $\vartheta$ is a $\sigma$-finite (positive) measure on $I_{1} \times I_{2}=$ $\left[a_{1}, b_{1}\right] \times\left[a_{2}, b_{2}\right] \subseteq \overline{\mathbb{R}}^{2}$ such that $G_{\vartheta}$ is finite on $\left[a_{1}, b_{1}\right) \times\left[a_{2}, b_{2}\right)$ and let $F_{1}, F_{2}$ be arbitrary continuous one-dimensional d.f. $s$ fulfilling $F_{i}\left(b_{i}\right)-F_{i}\left(a_{i}\right)=1$. Let $H^{*} \in \mathcal{F}_{F_{1}, F_{2}}$ be defined by $H^{*}=M \circ\left(F_{1}, F_{2}\right)$ and set $T^{*}:=F_{2}^{-} \circ F_{1}$. Then we have

$$
\begin{aligned}
\bar{m}_{G_{\vartheta}} & =\sup _{H \in \mathcal{F}\left(F_{1}, F_{2}\right)} \int_{I_{1} \times I_{2}} G_{\vartheta}(x, y) \mathrm{d} \mu_{H}(x, y)=\int_{I_{1} \times I_{2}} G_{\vartheta}(x, y) \mathrm{d} \mu_{H^{*}}(x, y) \\
& =\int_{I_{1}} G_{\vartheta}\left(x, T^{*}(x)\right) \mathrm{d} \xi_{F_{1}}(x) .
\end{aligned}
$$

Furthermore, the following two conditions are equivalent for every continuous $H \in \mathcal{F}\left(F_{1}, F_{2}\right)$ :

A) $\int_{I_{1} \times I_{2}} G_{\vartheta}(x, y) \mathrm{d} \mu_{H}(x, y)<\bar{m}_{G_{\vartheta}}$.

В) $\vartheta\left(\left\{(v, w) \in I_{1} \times I_{2}: H(v, w)<H^{*}(v, w)\right\}\right)>0$.

Proof. Fix $H \in \mathcal{F}\left(F_{1}, F_{2}\right)$ and let $A \in \mathcal{C}$ denote the corresponding copula fulfilling $H=A \circ\left(F_{1}, F_{2}\right)$. Setting $\Gamma:=\left\{(x, y, v, w) \in I_{1} \times I_{2} \times I_{1} \times I_{2}: v \leq\right.$ $x, w \leq y\}$ and letting $\mu_{H} \otimes \vartheta$ denote the product measure of $\mu_{H}$ and $\vartheta$, Fubini's theorem implies

$$
\mu_{H} \otimes \vartheta(\Gamma)=\int_{I_{1} \times I_{2}} \vartheta\left(\Gamma_{(x, y)}\right) \mathrm{d} \mu_{H}(x, y)=\int_{I_{1} \times I_{2}} G_{\vartheta}(x, y) \mathrm{d} \mu_{H}(x, y),
$$

where $\Gamma_{(x, y)}=\{(v, w):(x, y, v, w) \in \Gamma\}$ denotes the $(x, y)$-cut of $\Gamma$. Using the fact that $\Gamma_{(v, w)}=\left\{(x, y) \in I_{1} \times I_{2}:(x, y, v, w) \in \Gamma\right\}=\left[v, b_{1}\right] \times\left[w, b_{2}\right]$, again 


\section{F. DURANTE - J. FERNÁNDEZ-SÁNCHEZ - C. IGNAZZI - W. TRUTSCHNIG}

applying Fubini's theorem (this time in the other direction) and using continuity of $F_{1}, F_{2}$ and Sklar's theorem we get

$$
\begin{aligned}
\mu_{H} \otimes \vartheta(\Gamma) & =\int_{I_{1} \times I_{2}} \mu_{H}\left(\Gamma_{(v, w)}\right) \mathrm{d} \vartheta(v, w)=\int_{I_{1} \times I_{2}} \mu_{H}\left(\left[v, b_{1}\right] \times\left[w, b_{2}\right]\right) \mathrm{d} \vartheta(v, w) \\
& =\int_{I_{1} \times I_{2}}(1-F_{1}(v)-F_{2}(w)+\underbrace{A\left(F_{1}(v), F_{2}(w)\right)}_{\leq H^{*}(v, w)}) \mathrm{d} \vartheta(v, w) \\
& \leq \int_{I_{1} \times I_{2}} \mu_{H^{*}}\left(\Gamma_{(v, w)}\right) \mathrm{d} \vartheta(v, w)=\mu_{H^{*}} \otimes \vartheta(\Gamma)
\end{aligned}
$$

from which the first part of Eq. (2) follows immediately. Considering that

$$
K(x,(-\infty, y])=K_{M}\left(F_{1}(x),\left[0, F_{2}(y)\right]\right)=\mathbf{1}_{[0, y]}\left(T^{*}(x)\right)
$$

is a Markov kernel of $H^{*}$ (see [8]), the disintegration of the measure immediately yields

$$
\begin{aligned}
\int_{I_{1} \times I_{2}} G_{\vartheta}(x, y) \mathrm{d} \mu_{H^{*}}(x, y) & =\int_{I_{1}} \int_{I_{2}} G_{\vartheta}(x, y) K(x, \mathrm{~d} y) \mathrm{d} \xi_{F_{1}}(x) \\
& =\int_{I_{1}} G_{\vartheta}\left(x, T^{*}(x)\right) \mathrm{d} \xi_{F_{1}}(x),
\end{aligned}
$$

which completes the proof of Eq. (2).

Concerning the equivalence stated in the theorem we obviously have equality in (3) if, and only if,

$$
\int_{\left(a_{1}, b_{1}\right) \times\left(a_{2}, b_{2}\right)} A\left(F_{1}(v), F_{2}(w)\right) \mathrm{d} \vartheta(v, w)=\int_{\left(a_{1}, b_{1}\right) \times\left(a_{2}, b_{2}\right)} M\left(F_{1}(v), F_{2}(w)\right) \mathrm{d} \vartheta(v, w)
$$

holds, from which the result follows immediately.

Corollary 1. Under the assumptions of Theorem 1, Eq. (21) is also valid for discontinuous $F_{1}, F_{2}$.

P r o of. Using Eq. (3), considering

$$
\begin{aligned}
\mu_{H}\left(\left[v, b_{1}\right] \times\left[w, b_{2}\right]\right) & =\lim _{n \rightarrow \infty} \mu_{H}\left(\left(v-\frac{1}{n}, b_{1}\right] \times\left(w-\frac{1}{n}, b_{2}\right]\right) \\
& =\lim _{n \rightarrow \infty}\{1-F_{1}\left(v-\frac{1}{n}\right)-F_{2}\left(w-\frac{1}{n}\right)+\underbrace{H\left(v-\frac{1}{n}, w-\frac{1}{n}\right)}_{\leq H^{*}\left(v-\frac{1}{n}, w-\frac{1}{n}\right)}\} \\
& \leq \lim _{n \rightarrow \infty} \mu_{H^{*}}\left(\left(v-\frac{1}{n}, b_{1}\right] \times\left(w-\frac{1}{n}, b_{2}\right]\right) \\
& =\mu_{H^{*}}\left(\left[v, b_{1}\right] \times\left[w, b_{2}\right]\right)
\end{aligned}
$$

and proceeding as in the proof of Theorem 1, the desired inequality is obtained. 


\section{EXTREMAL PROBLEMS FOR PAIRS OF UNIFORMLY DISTRIBUTED SEQUENCES}

In case $F_{1}$ and $F_{2}$ are assumed to be continuous, all elements $H \in \mathcal{F}\left(F_{1}, F_{2}\right)$ are continuous too. Hence, if $H(v, w)<H^{*}(v, w)$ holds for some $v, w \in I_{1} \times I_{2}$, then it also holds in a neighbourhood of $(v, w)$. From this we directly get the following result.

COROLlaRY 2. If, under the assumption of Theorem 1, $\vartheta$ has full support, then $H^{*}$ is the unique element in $\mathcal{F}\left(F_{1}, F_{2}\right)$ attaining the maximum $\bar{m}_{G_{\vartheta}}$.

EXAMPle 1. Suppose that $I_{1}=I_{2}=[0,1]$, that $\vartheta$ is an arbitrary finite measure on $\left[0, \frac{3}{4}\right]^{2}$ and that $F_{1}(x)=F_{2}(x)=x \mathbf{1}_{[0,1]}(x)+\mathbf{1}_{(1, \infty)}(x)$. Furthermore let $H$ denote the ordinal sum of $M$ and $\Pi$ with respect to the partition $\left\{\left[0, \frac{3}{4}\right],\left[\frac{3}{4}, 1\right]\right\}$ (see [9]), i.e.,

$$
H(x, y)= \begin{cases}\frac{3}{4}+\frac{1}{4}(4 x-3)(4 y-3), & (x, y) \in[3 / 4,1]^{2} \\ \min (x, y), & \text { otherwise }\end{cases}
$$

Then, obviously, $\mathcal{F}\left(F_{1}, F_{2}\right)=\mathcal{C}$ and $\{(v, w): H(v, w) \neq M(v, w)\}=\left(\frac{3}{4}, 1\right)^{2}$. Since $\vartheta\left(\left(\frac{3}{4}, 1\right)^{2}\right)=0$ holds, according to Theorem 1 we have

$$
\bar{m}_{G_{\vartheta}}=\int_{[0,1]^{2}} G_{\vartheta} \mathrm{d} \mu_{H}=\int_{[0,1]^{2}} G_{\vartheta} \mathrm{d} \mu_{M},
$$

so the maximum $\bar{m}_{G_{\vartheta}}$ is attained by two different copulas, $M$ and $H$.

Example 2. Assuming, as in the previous example, $I_{1}=I_{2}=[0,1]$, let $G_{\vartheta}$ be a convex combination of $\Pi$ and $M$, that is, $G_{\vartheta}=\alpha \Pi+(1-\alpha) M$ for some $\alpha \in(0,1)$. First, observe that $\vartheta$ has full support, since

$$
\vartheta=\alpha \mu_{\Pi}+(1-\alpha) \mu_{M} \quad \text { and } \quad \mu_{\Pi}=\lambda_{2}
$$

has full support. So, in this case, according to Theorem 1 and Corollary 2, $M$ is the only copula attaining the maximum $\bar{m}_{G_{\vartheta}}$. Moreover,

$$
\begin{aligned}
\bar{m}_{G_{\vartheta}} & =\int_{[0,1]^{2}} \alpha \Pi+(1-\alpha) M \mathrm{~d} \mu_{M} \\
& =\alpha \int_{[0,1]} \Pi(t, t) \mathrm{d} \lambda(t)+(1-\alpha) \int_{[0,1]} M(t, t) \mathrm{d} \lambda(t) \\
& =\frac{\alpha}{3}+\frac{1-\alpha}{2}=\frac{1}{2}-\frac{\alpha}{6} .
\end{aligned}
$$

The following result provides a lower bound for integrals of type (2). It can be proved by mimicking the arguments presented in Theorem 1 . 


\section{F. DURANTE - J. FERNÁNDEZ-SÁNCHEZ - C. IGNAZZI - W. TRUTSCHNIG}

TheOREM 2. Suppose that $\vartheta$ is a $\sigma$-finite (positive) measure on

$$
I_{1} \times I_{2}=\left[a_{1}, b_{1}\right] \times\left[a_{2}, b_{2}\right] \subseteq \overline{\mathbb{R}}^{2}
$$

such that $G_{\vartheta}$ is finite on $\left[a_{1}, b_{1}\right) \times\left[a_{2}, b_{2}\right)$ and let $F_{1}, F_{2}$ be arbitrary continuous one-dimensional d.f.s fulfilling $F_{i}\left(b_{i}\right)-F_{i}\left(a_{i}\right)=1$. Let $H_{*} \in \mathcal{F}_{F_{1}, F_{2}}$ be defined by $H_{*}=W \circ\left(F_{1}, F_{2}\right)$ and set $T_{*}:=F_{2}^{-} \circ\left(1-F_{1}\right)$. Then we have

$$
\begin{aligned}
\underline{m}_{G_{\vartheta}} & =\inf _{H \in \mathcal{F}\left(F_{1}, F_{2}\right)} \int_{I_{1} \times I_{2}} G_{\vartheta}(x, y) \mathrm{d} \mu_{H}(x, y) \\
& =\int_{I_{1} \times I_{2}} G_{\vartheta}(x, y) \mathrm{d} \mu_{H_{*}}(x, y)=\int_{I_{1}} G_{\vartheta}\left(x, T_{*}(x)\right) \mathrm{d} \xi_{F_{1}}(x) .
\end{aligned}
$$

Furthermore, the following two conditions are equivalent for every continuous $H \in \mathcal{F}\left(F_{1}, F_{2}\right)$ :
A) $\int_{I_{1} \times I_{2}} G_{\vartheta}(x, y) \mathrm{d} \mu_{H}(x, y)>\underline{m}_{G_{\vartheta}}$.
В) $\vartheta\left(\left\{(v, w) \in I_{1} \times I_{2}: H(v, w)>H_{*}(v, w)\right\}\right)>0$.

Now, fix $y_{0} \in(0,1)$ be arbitrary and suppose both that $\vartheta_{1}$ is a finite (positive) measure on $[0,1] \times\left[0, y_{0}\right]$ and that $\vartheta_{2}$ is a finite (positive) measure on $[0,1] \times\left(y_{0}, 1\right]$. Setting $\vartheta=\vartheta_{1}-\vartheta_{2}$ yields a finite signed measure on $\mathcal{B}\left([0,1]^{2}\right)$ with Jordan decomposition $\vartheta_{1}-\vartheta_{2}$ (see [12]).

In the following we will consider the (measurable and bounded) function

$$
G_{\vartheta}(x, y)=\vartheta([0, x] \times[0, y]) .
$$

Slightly generalizing the results in [4, 6] we want to calculate

$$
\bar{m}_{G_{\vartheta}}=\sup _{A \in \mathcal{C}} \int_{[0,1]^{2}} G_{\vartheta} \mathrm{d} \mu_{A} .
$$

REMARK 1. Notice that, in [4, 6], instead of $G_{\vartheta}$, the integrand is assumed to be a continuous function $G$ on $[0,1]^{2}$ fulfilling $\frac{\partial^{2} G(x, y)}{\partial y \partial x}>0$ on $(0,1) \times\left(0, y_{0}\right)$ and $\frac{\partial^{2} G(x, y)}{\partial y \partial x}<0$ on $(0,1) \times\left(y_{0}, 1\right)$. Such a function can be considered as special cases of $G_{\vartheta}$. In fact, setting

$$
\begin{aligned}
& \vartheta_{1}(E \times F):=\int_{E \times\left(F \cap\left[0, y_{0}\right]\right)} \frac{\partial^{2} G(x, y)}{\partial y \partial x} \mathrm{~d} \lambda_{2}(x, y), \\
& \vartheta_{2}(E \times F):=\int_{E \times\left(F \cap\left(y_{0}, 1\right]\right)}-\frac{\partial^{2} G(x, y)}{\partial y \partial x} \mathrm{~d} \lambda_{2}(x, y),
\end{aligned}
$$

for $E, F \in \mathcal{B}([0,1])$ yields absolutely continuous, finite measures $\vartheta_{1}, \vartheta_{2}$. 


\section{EXTREMAL PROBLEMS FOR PAIRS OF UNIFORMLY DISTRIBUTED SEQUENCES}

For $\vartheta=\vartheta_{1}-\vartheta_{2}$ we obviously get $G_{\vartheta}=G+a$ for some constant $a \in \mathbb{R}$, implying

$$
\sup _{A \in \mathcal{C}} \int_{[0,1]^{2}} G_{\vartheta} \mathrm{d} \mu_{A}=\sup _{A \in \mathcal{C}} \int_{[0,1]^{2}} G \mathrm{~d} \mu_{A}+a .
$$

Our general setting also includes the results from [15, Theorems 28 and 29] and $[2]$.

As already shown in [4, Theorem 8] the problem of calculating $\bar{m}_{G_{\vartheta}}$ can be reduced to a one-dimensional maximization problem. Before restating Theorem 8 in [4] and proving it in an alternative and shorter way we define the class $\mathcal{H}_{y_{0}}$ as the set of all $y_{0}$-sections of copulas, i.e., the family of all the maps of the form $x \mapsto C\left(x, y_{0}\right), x \in[0,1], C \in \mathcal{C}$, and state some of its properties (see [7]). First, it is clear that each element $h \in \mathcal{H}_{y_{0}}$ has the following properties:

i) $h(0)=0, h(1)=y_{0}$,

ii) $h$ is non-decreasing and Lipschitz continuous (with Lipschitz constant $L=$ 1) and

iii) $h$ fulfils $h(x) \in\left[W\left(x, y_{0}\right), M\left(x, y_{0}\right)\right]$.

Conversely, it is straightforward to verify that each function $h:[0,1] \rightarrow\left[0, y_{0}\right]$ fulfilling properties (i)-(iii) is the $y_{0}$-section of a copula. In fact, the function $C_{h}$, defined by

$$
C_{h}(x, y)=\left\{\begin{aligned}
M(h(x), y) & \text { if }(x, y) \in[0,1] \times\left[0, y_{0}\right], \\
h(x)+\left(1-y_{0}\right) W\left(\frac{x-h(x)}{1-y_{0}}, \frac{y-y_{0}}{1-y_{0}}\right) & \text { if }(x, y) \in[0,1] \times\left(y_{0}, 1\right] .
\end{aligned}\right.
$$

is easily shown to be a copula whose $y_{0}$-section coincides with $h$. Additionally, setting $\bar{h}(x)=1-(x-h(x))$ for every $x \in[0,1]$ as well as

$$
K_{C_{h}}(x, E)=\mathbf{1}_{E}(h(x)) h^{\prime}(x)+\mathbf{1}_{E}(\bar{h}(x))\left(1-h^{\prime}(x)\right)
$$

for $E \in \mathcal{B}([0,1])$ and for every $x \in[0,1]$ at which $h$ is differentiable (recall that $h$ is differentiable at $\lambda$-a.e. $x \in[0,1])$, it is straightforward to verify that $K_{C_{h}}$ is a Markov kernel of $C_{h}$ (see, e.g., [16]) and that $C_{h}$ concentrates its mass on $\Gamma(h) \cup \Gamma(\bar{h})$ (where $\Gamma(h)$ and $\Gamma(\bar{h})$ denote the graphs of $h$ and $\bar{h}$, respectively) in the sense that $\mu_{C_{h}}(\Gamma(h) \cup \Gamma(\bar{h}))=1$.

Theorem 3. Consider $y_{0} \in(0,1)$ and suppose that $\vartheta_{1}$ is a finite (positive) measure on $[0,1] \times\left[0, y_{0}\right]$ and that $\vartheta_{2}$ is a finite (positive) measure on $[0,1] \times\left(y_{0}, 1\right]$. Set $\vartheta=\vartheta_{1}-\vartheta_{2}$ and consider the (measurable and bounded) function

$$
G_{\vartheta}(x, y)=\vartheta([0, x] \times[0, y]) .
$$




\section{F. DURANTE - J. FERNÁNDEZ-SÁNCHEZ - C. IGNAZZI - W. TRUTSCHNIG}

The following equality holds:

$$
\begin{aligned}
\sup _{B \in \mathcal{C}} \int_{[0,1]^{2}} G_{\vartheta} \mathrm{d} \mu_{B}= & \sup _{h \in \mathcal{H}_{y_{0}}} \int_{[0,1]^{2}} G_{\vartheta} \mathrm{d} \mu_{C_{h}} \\
= & \sup _{h \in \mathcal{H}_{y_{0}}} \int_{[0,1]} G_{\vartheta}(x, h(x)) h^{\prime}(x) \\
& +G_{\vartheta}(x, \bar{h}(x))\left(1-h^{\prime}(x)\right) \mathrm{d} \lambda(x) .
\end{aligned}
$$

Moreover, the former upper bound is attained (i.e., is a maximum) if and only if the latter is attained.

Proof. Let $A \in \mathcal{C}$ and set $h(x)=A\left(x, y_{0}\right)$ for every $x \in[0,1]$ and define two new (conditional) probability measures $\mu^{+}, \mu^{-}$by

$$
\begin{aligned}
& \mu^{+}(\Omega)=\frac{1}{y_{0}} \mu_{A}\left(\Omega \cap\left([0,1] \times\left[0, y_{0}\right]\right)\right), \\
& \mu^{-}(\Omega)=\frac{1}{1-y_{0}} \mu_{A}\left(\Omega \cap\left([0,1] \times\left[y_{0}, 1\right]\right)\right) \quad \text { for every } \Omega \in \mathcal{B}\left([0,1]^{2}\right) .
\end{aligned}
$$

Calculating both marginals of $\mu^{+}$and applying Theorem 1 directly yields $T^{*}=h$ as well as

$$
\int_{[0,1] \times\left[0, y_{0}\right]} G_{\vartheta} \mathrm{d} \mu^{+} \leq \frac{1}{y_{0}} \int_{[0,1]} G_{\vartheta}(x, h(x)) h^{\prime}(x) \mathrm{d} \lambda(x) .
$$

Considering that for every $y \in\left(y_{0}, 1\right]$ we have

$$
G_{\vartheta}(x, y)=G_{\vartheta}\left(x, y_{0}\right)-\vartheta_{2}\left([0, x] \times\left[y_{0}, y\right]\right)
$$

and setting

$$
I=\int_{[0,1] \times\left[y_{0}, 1\right]} G_{\vartheta}(x, y) \mathrm{d} \mu^{-}(x, y)
$$

we get

$$
\begin{aligned}
I & =\int_{[0,1] \times\left[y_{0}, 1\right]} G_{\vartheta}\left(x, y_{0}\right) \mathrm{d} \mu^{-}(x, y)-\int_{[0,1] \times\left[y_{0}, 1\right]} \vartheta_{2}\left([0, x] \times\left[y_{0}, y\right]\right) \mathrm{d} \mu^{-}(x, y) \\
& =\frac{1}{1-y_{0}} \int_{[0,1]} G_{\vartheta}\left(x, y_{0}\right)\left(1-h^{\prime}(x)\right) \mathrm{d} \lambda(x)-\underbrace{\int_{[0,1] \times\left[y_{0}, 1\right]} \vartheta_{2}\left([0, x] \times\left[y_{0}, y\right]\right) \mathrm{d} \mu^{-}(x, y)}_{I^{*}},
\end{aligned}
$$

whereby the last equality follows from the disintegration of a measure and the fact that we have

$$
K_{A}\left(x,\left(y_{0}, 1\right]\right)=1-h^{\prime}(x) \quad \text { for } \quad \lambda \text {-a.e. } x \in[0,1] .
$$




\section{EXTREMAL PROBLEMS FOR PAIRS OF UNIFORMLY DISTRIBUTED SEQUENCES}

Calculating the marginals of $\mu^{-}$and applying Theorem 2 to $I^{*}$ we get $T_{*}=\bar{h}$ as well as

$$
I^{*} \geq \frac{1}{1-y_{0}} \int_{[0,1]} \vartheta_{2}\left([0, x] \times\left[y_{0}, \bar{h}(x)\right]\right)\left(1-h^{\prime}(x)\right) \mathrm{d} \lambda(x),
$$

so, altogether

$$
\begin{aligned}
I \leq & \frac{1}{1-y_{0}}\left(\int_{[0,1]} G_{\vartheta}\left(x, y_{0}\right)\left(1-h^{\prime}(x)\right) \mathrm{d} \lambda(x)-\right. \\
& \left.\int_{[0,1]} \vartheta_{2}\left([0, x] \times\left[y_{0}, \bar{h}(x)\right]\right)\left(1-h^{\prime}(x)\right) \mathrm{d} \lambda(x)\right) \\
= & \frac{1}{1-y_{0}} \int_{[0,1]} G_{\vartheta}(x, \bar{h}(x))\left(1-h^{\prime}(x)\right) \mathrm{d} \lambda(x) .
\end{aligned}
$$

Considering $\mu_{A}=y_{0} \mu^{+}+\left(1-y_{0}\right) \mu^{-}$we conclude that the first quantity in Eq. (8) cannot be greater than the third one. Since, as direct consequence of Eq. (7), we have

$$
\begin{aligned}
\int_{[0,1]^{2}} G_{\vartheta} \mathrm{d} \mu_{C_{h}}= & \int_{[0,1]} G_{\vartheta}(x, h(x)) h^{\prime}(x) \\
& +G_{\vartheta}(x, \bar{h}(x))\left(1-h^{\prime}(x)\right) \mathrm{d} \lambda(x)
\end{aligned}
$$

and since $C_{h}$ is a copula with $y_{0}$-section $h$, the proof of Eq. (8) is complete.

In general it seems unknown if, under the assumptions of Theorem 3 , there is a unique function $h \in \mathcal{H}_{y_{0}}$ attaining the maximum. In special cases, uniqueness is clear - in the following we consider a slightly more general version of Example 3 in [4] and show uniqueness directly (without Euler equation).

Example 3. As before suppose that $y_{0} \in(0,1)$. Let $\vartheta_{1}$ and $\vartheta_{2}$ denote absolutely continuous measures with constant densities $a>0$ on the rectangle $[0,1] \times\left[0, y_{0}\right]$ and $b>0$ on $[0,1] \times\left(y_{0}, 1\right]$, respectively, and set

$$
\vartheta:=\vartheta_{1}-\vartheta_{2} .
$$

The corresponding function $G_{\vartheta}$ is given by

$$
G_{\vartheta}(x, y)=\left\{\begin{aligned}
a x y & \text { if }(x, y) \in[0,1] \times\left[0, y_{0}\right], \\
a x y_{0}-b x\left(y-y_{0}\right) & \text { if }(x, y) \in[0,1] \times\left(y_{0}, 1\right] .
\end{aligned}\right.
$$




\section{F. DURANTE - J. FERNÁNDEZ-SÁNCHEZ - C. IGNAZZI - W. TRUTSCHNIG}

For arbitrary $h \in \mathcal{H}_{y_{0}}$ applying Theorem 3 and using integration by parts we get

$$
\begin{aligned}
\int_{[0,1]^{2}} G_{\vartheta} \mathrm{d} \mu_{C_{h}}= & a \int_{[0,1]} x h(x) h^{\prime}(x) \mathrm{d} \lambda(x) \\
& +\int_{[0,1]} x\left[(a+b) y_{0}-b+b x-b h(x)\right]\left(1-h^{\prime}(x)\right) \mathrm{d} \lambda(x) \\
= & \frac{a}{2}\left\{\left.x h^{2}(x)\right|_{0} ^{1}-\int_{[0,1]} h^{2}(x) \mathrm{d} \lambda(x)\right\} \\
& +\frac{1}{2 b}\left\{\left.x\left[(a+b) y_{0}-b+b x-b h(x)\right]^{2}\right|_{0} ^{1}\right. \\
& \left.-\int_{[0,1]}\left[(a+b) y_{0}-b+b x-b h(x)\right]^{2} \mathrm{~d} \lambda(x)\right\} \\
= & \frac{a}{2} y_{0}^{2}-\frac{a}{2} \int_{[0,1]} h^{2}(x) \mathrm{d} \lambda(x)+\frac{a^{2}}{2 b} y_{0}^{2} \\
& -\int_{[0,1]} \underline{\left[(a+b) y_{0}-b+b x-b h(x)\right]^{2}} \mathrm{~d} \lambda(x) \\
= & \left(\frac{a}{2}+\frac{a^{2}}{b}\right) y_{0}^{2} \\
& -\frac{1}{2 b} \int_{[0,1]}\left(a b+b^{2}\right) h^{2}(x) \\
& -2 b\left[(a+b) y_{0}-b+b x\right] h(x) \mathrm{d} \lambda(x) \\
& {\left[(a+b) y_{0}-b+b x\right]^{2} \mathrm{~d} \lambda(x) . } \\
& \\
& \\
& \\
& \\
&
\end{aligned}
$$

For fixed $x$ the latter integrand becomes minimal if

The function

$$
h(x):=y_{0}+\frac{b}{a+b}(x-1) .
$$

$$
h_{1}: x \mapsto y_{0}+\frac{b}{a+b}(x-1)
$$

is a global minimizer of the integral which, however, only lies in

$$
\mathcal{H}_{y_{0}} \text { for } \quad y_{0}=\frac{b}{a+b} .
$$




\section{EXTREMAL PROBLEMS FOR PAIRS OF UNIFORMLY DISTRIBUTED SEQUENCES}

It is straightforward to verify that for $y_{0} \geq \frac{b}{a+b}$ the (piecewise linear) function $h$, defined by

$$
h(x):=\left\{\begin{aligned}
x & \text { if } x \in\left[0, \frac{(a+b) y_{0}-b}{a}\right], \\
y_{0}+\frac{b}{a+b}(x-1) & \text { if } x \in\left(\frac{(a+b) y_{0}-b}{a}, 1\right]
\end{aligned}\right.
$$

is the best approximation of $h_{1}$ in $\mathcal{H}_{y_{0}}$. Figure 1 depicts a sample of the corresponding copula $C_{h}$ for the case $a=b=1$ and $y_{0}=\frac{3}{4}$.

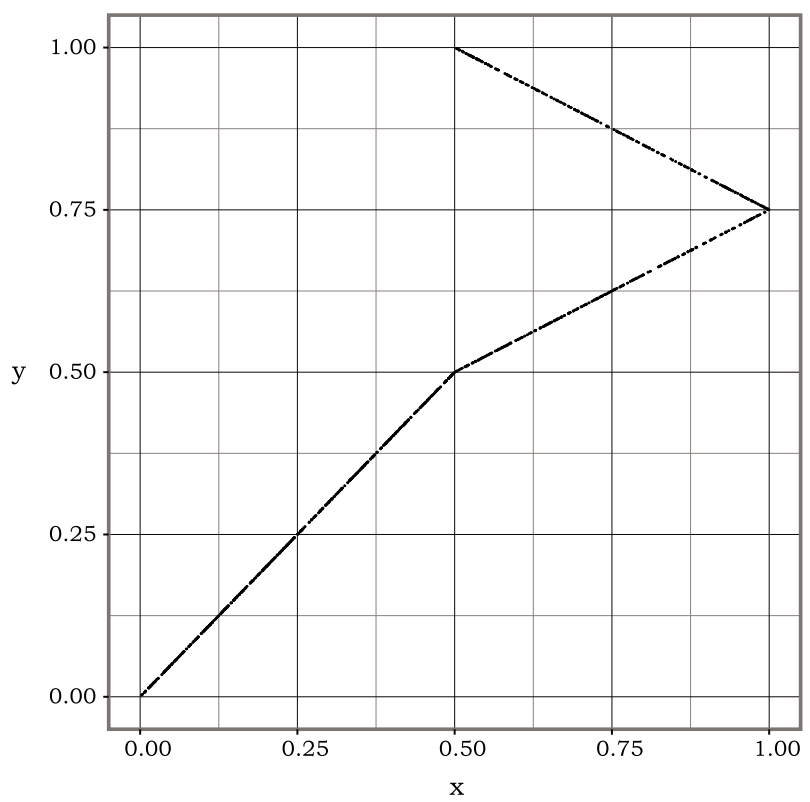

FIGURE 1.

Sample of size $n=1.000$ of the unique maximizer $C_{h}$ in Example 3 for the case $a=b=1$ and $y_{0}=\frac{3}{4}$.

Finally, we aim at showing that every $h \in \mathcal{H}_{y_{0}}$ is in fact a possible maximizer in the sense that there exists some $\vartheta=\vartheta_{1}-\vartheta_{2}$ such that

$$
\sup _{B \in \mathcal{C}} \int_{[0,1]^{2}} G_{\vartheta} \mathrm{d} \mu_{B}=\int_{[0,1]^{2}} G_{\vartheta} \mathrm{d} \mu_{C_{h}} .
$$




\section{F. DURANTE - J. FERNÁNDEZ-SÁNCHEZ - C. IGNAZZI - W. TRUTSCHNIG}

ThEOREM 4. For every $h \in \mathcal{H}_{y_{0}}$ there exists a measure $\vartheta$ on $\mathcal{B}\left([0,1]^{2}\right)$ such that

$$
\sup _{B \in \mathcal{C}} \int_{[0,1]^{2}} G_{\vartheta} \mathrm{d} \mu_{B}=\int_{[0,1]^{2}} G_{\vartheta} \mathrm{d} \mu_{C_{h}}
$$

P r o of. Fix $h \in \mathcal{H}_{y_{0}}$ and let $\vartheta$ denote the (singular) probability measure spreading its mass uniformly on $\Gamma(h)$. Thus, for every $x \in[0,1]$, we have

$$
G_{\vartheta}(x, h(x))=x,
$$

and on the other hand,

$$
G_{\vartheta}(x, \bar{h}(x))=G_{\vartheta}\left(x, y_{0}\right)=x
$$

as well. Hence

$$
\int_{[0,1]^{2}} G_{\vartheta} \mathrm{d} \mu_{C_{h}}=\int_{[0,1]} x \mathrm{~d} \lambda(x)=\frac{1}{2} .
$$

Let us now consider an arbitrary $h^{*} \in \mathcal{H}_{y_{0}}$, and let $x \in[0,1]$ be any point at which both $h$ and $h^{*}$ are differentiable. If $h^{*}(x)<h(x)$ holds, then obviously,

$$
G_{\vartheta}\left(x, h^{*}(x)\right)<x
$$

whereas

$$
G_{\vartheta}\left(x, \overline{h^{*}}(x)\right)=G_{\vartheta}\left(x, y_{0}\right)=x,
$$

so for those values of $x$ one has

$$
G_{\vartheta}\left(x, h^{*}(x)\right) \frac{\mathrm{d} h^{*}}{\mathrm{~d} x}(x)+G_{\vartheta}\left(x, \overline{h^{*}}(x)\right)\left(1-\frac{\mathrm{d} h^{*}}{\mathrm{~d} x}(x)\right) \leq x,
$$

where the equality holds if and only if $\frac{\mathrm{d} h^{*}}{\mathrm{~d} x}(x)=0$. On the other hand, if $h^{*}(x)>h(x)$ holds, then it is easily checked that

$$
G_{\vartheta}\left(x, h^{*}(x)\right)=G_{\vartheta}\left(x, \overline{h^{*}}(x)\right)=x,
$$

so for those values of $x$ one has

$$
G_{\vartheta}\left(x, h^{*}(x)\right) \frac{\mathrm{d} h^{*}}{\mathrm{~d} x}(x)+G_{\vartheta}\left(x, \overline{h^{*}}(x)\right)\left(1-\frac{\mathrm{d} h^{*}}{\mathrm{~d} x}(x)\right)=x .
$$

This proves that the copula $C_{h}$ associated to $h$ is a maximizer of $\int_{[0,1]^{2}} G_{\vartheta} \mathrm{d} \mu_{B}$ over all $B \in \mathcal{C}$, and the maximum is equal to $\frac{1}{2}$. 


\section{EXTREMAL PROBLEMS FOR PAIRS OF UNIFORMLY DISTRIBUTED SEQUENCES}

Acknowledgements. The first author has been supported by the project "Stochastic Models for Complex Systems" by Italian MIUR (PRIN 2017, Project no. 2017JFFHSH). The fourth author gratefully acknowledges the support of the WISS 2025 project 'IDA-lab Salzburg' (20204-WISS/225/197-2019 and 20102-F1901166-KZP).

\section{REFERENCES}

[1] AmBrosio, L.-GIGLI, N.: A user's guide to optimal transport. In: Modelling and Optimisation of Flows on Networks, Lecture Notes in Math. Vol. 2062, Springer, Heidelberg, 2013, pp. 1-155.

[2] BALÁŽ, V.-IACÒ, M. R.-STRAUCH, O.-THONHAUSER, S.-TICHY, R. F.: An extremal problem in uniform distribution theory, Unif. Distrib. Theory 11 (2016), no. 2. $1-21$.

[3] DURAnTE, F.-SEMPI, C.: Principles of Copula Theory, CRC Press, Boca Raton, FL, 2016.

[4] FIALOVÁ, J.-STRAUCH, O.: On two-dimensional sequences composed by onedimensional uniformly distributed sequences, Unif. Distrib. Theory 6 (2011), no. 1. $101-125$.

[5] HOFER, M.-IACÒ, M.R.: Optimal bounds for integrals with respect to copulas and applications, J. Optim. Theory Appl. 161 (2014), no. 3, 999-1011.

[6] IACÒ, M. R.-THONHAUSER, S.-TICHY, R. F.: Distribution functions, extremal limits and optimal transport, Indag. Math. (N.S.) 26 (2015), no. 5, 823-841.

[7] KLEMENT, E. P.-KOLESÁROVÁ, A.-MESIAR, R.-SEMPI, C.: Copulas constructed from horizontal sections, Comm. Statist. Theory Methods 36 (2007), 2901-2911.

[8] MROZ, T.-TRUTSCHNiG, W.-FERNÁNDEZ SÁNCHEZ, J.: Distributions with fixed marginals maximizing the mass of the endograph of a function, 2016. arXiv:1602.05807,

[9] NELSEN, R. B.: An Introduction to Copulas. 2nd edition. Springer Series in Statistics. Springer, New York, 2006.

[10] PILliChSHAMMER, F.-STEINERBERGER, S.: Average distance between consecutive points of uniformly distributed sequences. Unif. Distrib. Theory 4 (2009), no. 1, 51-67.

[11] RACHEV, S. T.-RÜSCHENDORF, L.: Mass Transportation Problems. Vol. I. Theory. Probability and its Applications (New York). Springer-Verlag, New York, 1998.

[12] RUDIN, W.: Real and Complex Analysis. 3rd ed. McGraw-Hill Book Co., New York, 1987.

[13] RÜSCHENDORF, L.: Mathematical Risk Analysis. In: Springer Series in Operations Research and Financial Engineering. Springer, Heidelberg, 2013.

[14] SANTAMBROGIO, F.: Optimal Transport for Applied Mathematicians. Calculus of Variations, PDEs, and Modeling. In:Progress in Nonlinear Differential Equations and their Applications Vol. 87. Birkhäuser/Springer, Cham, 2015. 


\section{F. DURANTE - J. FERNÁNDEZ-SÁNCHEZ - C. IGNAZZI - W. TRUTSCHNIG}

[15] STRAUCH, O.: Some applications of distribution functions of sequences, Unif. Distrib. Theory 10 (2015), no. 2, 117-183.

[16] TRUTSCHNIG, W.: On a strong metric on the space of copulas and its induced dependence measure, J. Math. Anal. Appl. 384 (2011), 690-705.

Received June 12, 2020

Accepted November 15, 2020

\section{Fabrizio Durante}

Dipartimento di Scienze dell'Economia Università del Salento

Campus Ecotekne - Palazzina C

Via Monteroni 165, I-73100 Lecce

ITALY

E-mail: fabrizio.durante@unisalento.it

\section{Juan Fernández Sánchez}

Grupo de investigación de Teoría de Cópulas y Aplicaciones

Universidad de Almería

Carretera Sacramento $s / n$

E-04120 La Cañada de San Urbano, Almería SPAIN

E-mail: juanfernandez@ual.es

\section{Claudio Ignazzi}

Dipartimento di Matematica e Fisica

"Ennio De Giorgi"

Università del Salento

via per Arnesano, I-73100 Lecce

ITALY

E-mail: claudio.ignazzi@unisalento.it

\section{Wolfgang Trutschnig}

Department for Mathematics

University of Salzburg

Hellbrunner Strasse 34, A-5020 Salzburg

AUSTRIA

E-mail: wolfgang@trutschnig.net 